Musées, Patrimoine et Culture scientifiques et techniques

$122 \mid 2009$

mars - avril 2009

\title{
Évaluer les bénéfices d'une activité muséale
}

Jessica Degain et Sophie Benharkate

URL : http://journals.openedition.org/ocim/288

DOI : $10.4000 /$ ocim.288

ISSN : 2108-646X

Éditeur

OCIM

Édition imprimée

Date de publication : 1 mars 2009

Pagination : 19-25

ISSN : 0994-1908

Référence électronique

Jessica Degain et Sophie Benharkate, «Évaluer les bénéfices d'une activité muséale », La Lettre de I'OCIM [En ligne], 122 | 2009, mis en ligne le 01 mars 2011, consulté le 01 mai 2019. URL : http:// journals.openedition.org/ocim/288; DOI : 10.4000/ocim.288 


\title{
Évaluer les bénéfices d'une activité muséale
}

\author{
Jessica Degain et Sophie Benharkate *
}

\author{
Comment mesurer le réel apport pour \\ le public d'une activité muséale? Pour \\ répondre à cette interrogation, les auteurs \\ présentent un instrument de recherche qui \\ permet aux professionnels des musées \\ d'évaluer - à partir de l'observation \\ comportementale - les retombées positives \\ pour les participants des programmes \\ éducatifs et culturels muséaux.
}

Depuis la seconde moitié du XXe siècle, le public se trouve résolument au cœur des préoccupations des musées. En témoignent la généralisation des services éducatifs et de médiation, ainsi que la multiplication des activités culturelles proposées. Des visites-conférences aux ateliers pédagogiques, la programmation éducative et culturelle des musées vise en effet à satisfaire un public le plus large possible. Il existe ainsi aujourd'hui une très grande diversité en matière de programmes éducatifs et culturels muséaux, qui varient selon la nature de l'activité proposée, le moment de son déroulement, le public concerné et son niveau d'expertise.

* Jessica Degain est doctorante à l'École du Louvre et au laboratoire du Centre de Recherches sur les Liens Sociaux (CERLIS), université Paris V René Descartes. jessica.degain@hotmail.com

Sophie Benharkate est étudiante en Master II Médiation culturelle à l'École du Louvre, et Métiers du patrimoine

à l'université de Saint-Étienne. sophie.benharkate@neuf.fr
Face à ce foisonnement, le souci d'évaluer ces activités a progressivement émergé au sein des services des publics, jusqu'à y occuper une place prépondérante. Les expositions temporaires, qui constituent la programmation la plus visible et la plus mobilisatrice des musées, ont de ce fait été les premières concernées par cette démarche évaluatrice. Tant et si bien que les études de réception des expositions temporaires sont aujourd'hui une pratique incontournable et bien rôdée dans les institutions qui les produisent.

On ne saurait cependant en dire autant des autres programmes éducatifs et culturels muséaux. Les ateliers, en particulier, sont encore loin de faire l'objet d'évaluations scientifiques systématiques. La revue des écrits et des pratiques montre d'ailleurs qu'il n'existe à ce jour - du moins à notre connaissance aucun outil reconnu permettant d'évaluer ce type de programme ${ }^{(1)}$.

Et pourtant, l'évaluation de tout ce qu'un musée produit est le garant de son bon fonctionnement et de sa vitalité. C'est dans cette optique que nous avons conçu un instrument permettant à des professionnels de musées d'évaluer les retombées positives de tout type de programme éducatif et culturel (à l'exception des expositions temporaires), c'est-à-dire les bénéfices qu'en retirent les participants. Nous verrons que cet instrument a aussi vocation à guider les professionnels dans la phase de conception d'un programme ${ }^{(2)}$.

Pour ce faire, nous commencerons par définir précisément les termes clés de "bénéfices » et d' "évaluation », avant de signaler la principale source d'inspiration de cet instrument. Ce dernier sera ensuite présenté en détail, au même titre que ses modalités de validation. Enfin, nous terminerons 
cette présentation de l'instrument en attirant l'attention du lecteur sur les atouts et limites inhérents à son emploi.

\section{Quelques précisions sémantiques}

Dans les lignes qui précédent, nous avons annoncé avoir créé un instrument permettant d'évaluer les retombées positives ou «bénéfices » d’un programme éducatif et culturel. Mais qu'entend-t-on exactement par «bénéfices »? Le dictionnaire Le Petit Larousse nous apprend que la racine latine du mot, beneficium, signifie «bienfait ». Dans le langage courant, c'est l'acception économique du terme qui prévaut. Parler de bénéfice revient en effet le plus souvent à considérer la notion sous l'angle de « produit financier réalisé par une entreprise, un commerce... grâce à son activité ». Pourtant, le terme de bénéfice peut aussi s'entendre de façon plus large comme étant un «avantage, un bienfait tiré de ». En muséologie, quelques chercheurs ont œuvré à l'élaboration d'une définition du concept de « bénéfices muséaux ». Dans leur ouvrage Psychologie du visiteur. Contribution à l'éducation des adultes en milieu muséal, Colette Dufresne-Tassé et André Lefebvre en donnent la définition suivante : "c’est le produit d'une visite, ce qu'elle rapporte, c'est-à-dire les fruits, les gains, les profits qu'elle occasionne et la satisfaction que ceux-ci entraînent» (1996, p. 110). C'est cette définition que nous retiendrons dorénavant lorsqu'il sera question des bénéfices retirés par les participants à une activité qu'il s'agit d'évaluer. Il convient cependant de noter que la littérature produite autour de la question des bénéfices muséaux ne prend en compte qu'une seule dimension : celle des visites individuelles d'expositions permanentes. À l'inverse, aucun ouvrage n'a semble-t-il encore traité des bénéfices retirés de la participation à un programme éducatif et culturel, du moins sous l'angle de ses modalités d'évaluation. D'où l'intérêt, en tant que chercheurs de s'y atteler.

Vouloir évaluer les bénéfices d'une activité requiert aussi que l'on définisse au préalable le terme « évaluation ». Cette démarche consiste à s'assurer que les objectifs que l'on s'est fixés ont bien été atteints. L'évaluation passe par l'analyse des caractéristiques de chaque composante du programme, ou si l'on préfère de l'ensemble des activités qui le constituent. Il s'agit en d'autres termes de voir jusqu'à quel point chaque activité correspond aux objectifs de départ ; avant de procéder à l'analyse des résultats de chaque activité, et des résultats dans leur globalité.
C'est précisément ce que nous avons cherché à faire au travers de la série de schémas constituant notre instrument.

\section{Présentation de l'instrument et sources $d$ 'inspiration}

Après une lecture attentive des différents écrits relatifs à la notion de bénéfices muséaux (Dufresne-Tassé, Lapointe, Lefebvre, 1993, pp. 1-28 ; Dufresne-Tassé, Lefebvre, 1996, pp. 109-129 ; Lefebvre, 1996, pp. 193205), nous avons conçu une série de schémas permettant d'évaluer les bénéfices d'une activité, en termes de développement de la personne. Cet instrument est donc le fruit de nos lectures, mais aussi d'une réflexion sur les conditions d'apparition et d'observation des bénéfices chez des participants.

Le travail de la chercheuse en muséologie MarieClarté O'Neill, nous a, à ce titre, été d'une aide très précieuse. Marie-Clarté O’Neill a en effet beaucoup œuvré en matière d'élaboration et d'évaluation de programmes éducatifs en milieu muséal. Le fruit de son travail a été présenté dans le cadre d'un séminaire de l'École du Louvre intitulé « Programmes éducatifs et médiation : l'accompagnement des publics » (janvier-avril 2007) (3). L'enjeu de ce séminaire était de concilier une approche à la fois théorique et méthodologique "permettant la caractérisation précise des produits offerts et l'analyse critique des méthodes utilisées ». À cette fin, Marie-Clarté O’Neill a proposé plusieurs schémas décortiquant les éléments et étapes d'une programmation, leur articulation et la construction d'une intervention au sein d'un programme. L'intérêt d'une telle démarche était double. Premièrement, optimiser la création d'un nouveau programme et deuxièmement, évaluer un programme éducatif déjà existant. Cette façon de procéder nous a guidées dans l'élaboration de notre propre instrument de recherche.

\section{Description de l'instrument}

Ce dernier, rappelons-le, permet d'évaluer les bénéfices de tout type de programme éducatif et culturel, quel que soit le public concerné, à partir de l'observation comportementale. Il a vocation à être utilisé par des professionnels de musées, soit en amont de la conception du programme, soit en aval pour vérifier la présence de bénéfices.

Cet instrument se compose de quatre schémas, allant du général au particulier. Le schéma principal 
$\left(n^{\circ} 4\right)$ étant l'aboutissement d'une série d'autres, comme l'indique le graphique de synthèse ci-dessous :



- le schéma 1, présente les étapes préalables qu’un professionnel doit respecter pour élaborer un programme générant des bénéfices.

- le schéma 2, réunit les conditions physiques et intellectuelles nécessaires à l'émergence de bénéfices chez les participants.

- le schéma 3, se place du point de vue du participant, et envisage l'impact des stimuli de l'activité sur ce dernier pour qu'apparaissent des bénéfices.

- enfin, le schéma 4 constitue l'instrument de recherche à proprement parler, permettant l'évaluation des bénéfices d'un programme sur le terrain.

Schéma 1 : Étapes préalables à l'élaboration ou à l'évaluation d'un programme générant des bénéfices



Ce schéma représente les différentes étapes que doit respecter un professionnel pour s'assurer que le programme créé, ou à créer, va engendrer les bénéfices escomptés. En premier lieu, il convient de définir très clairement les objectifs du programme [1]. En second lieu, il est important de définir le public ciblé et d'en étudier les caractéristiques[2-3]. Notons que cette étape peut précéder la définition des objectifs : pour la conception d'un programme, on peut soit partir du public, soit partir d'objectifs. Découle de ces trois premières étapes la définition des bénéfices visés [4]. Il est alors important de savoir ce qui se fait, ou ce qui s'est fait, de comparable au programme dans d'autres institutions [5]. Ceci dans le but de s'en inspirer, de l'adapter ou de le dépasser. Une fois que l'on est parvenu à se faire une idée précise du programme, il faut tenir compte des moyens humains, financiers, spatio-temporels et matériels dont on dispose [6]. Au regard des étapes précédentes, on sera enfin en mesure de proposer un programme adapté - c'est-à-dire doté d'objectifs, d'un public cible, de moyens d'action, de conditions d'application et de mise en œuvre clairement définis - qui engendrera des bénéfices. Si l'activité est déjà créée, il s'agira de vérifier qu'il y a une bonne adéquation entre les objectifs, le public ciblé et les bénéfices escomptés[7-8]. Ceci n’est pas à négliger, sous peine de passer à côté de potentiels dysfonctionnements, qui auront d'évidentes répercussions sur le déroulement de l'activité et les bénéfices qu'en retireront les participants.

\section{Schéma 2 : Conditions physiques ef} intellectuelles à respecter pour favoriser l'émergence de bénéfices chez les participants

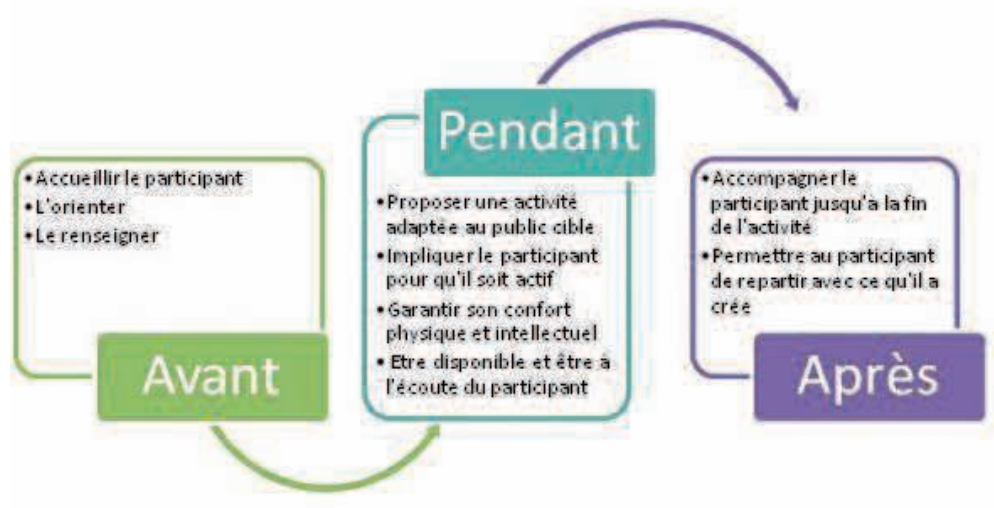

Pour favoriser l'émergence de bénéfices chez les participants, un certain nombre de conditions sont à respecter selon les différents moments du programme. 
Avant le commencement de l'activité, il est important que le participant soit bien accueilli, orienté et renseigné sur le contenu du programme. C'est à ces conditions que le visiteur se sentira sécurisé. Pendant, une activité adaptée au public ciblé permettra d'optimiser les conditions physiques et intellectuelles qui conduiront à l'apparition de bénéfices. L'encadrement devra être optimal : l'animateur se doit d'être disponible, à l'écoute et d'impliquer les participants. Il est également nécessaire de garantir leur confort physique et intellectuel.

Après, l'accompagnement des participants devra se faire jusqu'à ce que ces derniers quittent la structure, avec - le cas échéant - l'objet qu'ils ont créé durant l'activité.

Ces recommandations, une fois énoncées, peuvent sembler aller de soi. Pourtant, l'observation de plusieurs programmes éducatifs et culturels au sein de divers musées nous a prouvé que ces principes n'étaient pas toujours respectés. D'où l'utilité de les rappeler ici à l'esprit des concepteurs et animateurs de tels programmes.

Schéma 3 : Impact des stimuli de l'activité sur le participant en termes de bénéfices

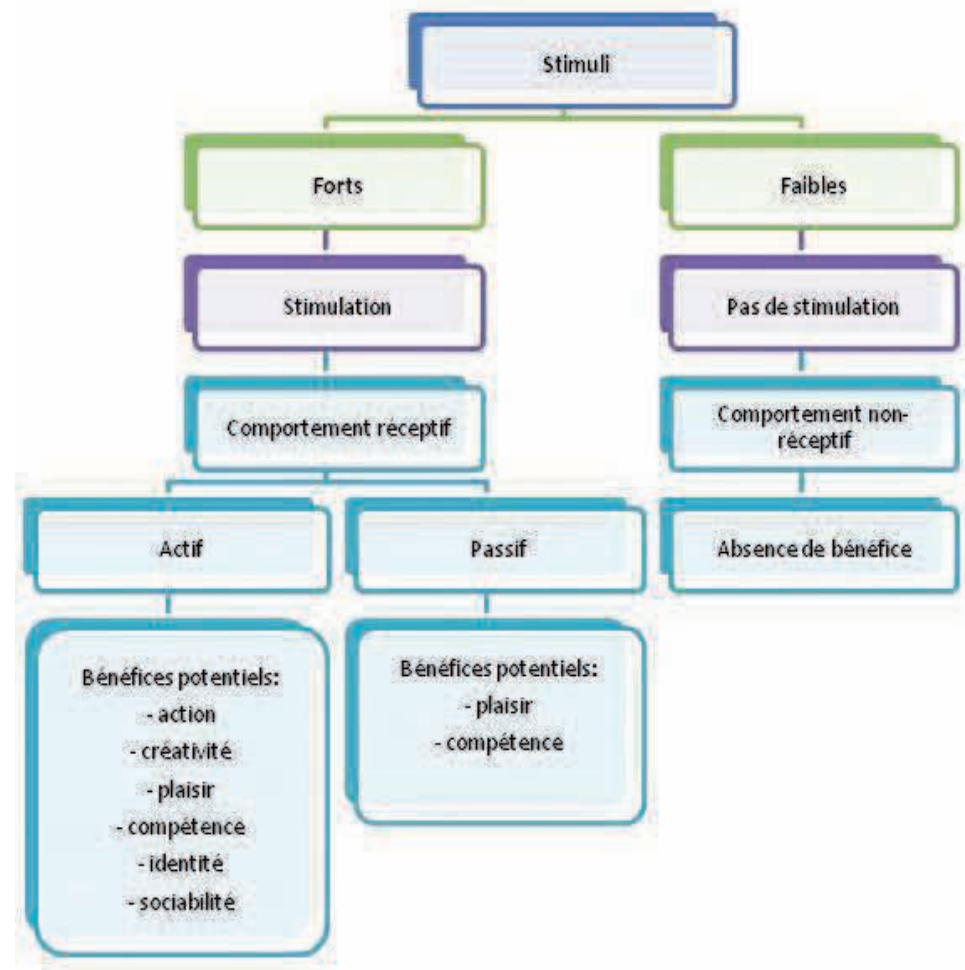

Avec ce troisième schéma, nous envisageons les bénéfices d'une activité sous un angle plus théorique. En considérant un programme éducatif et culturel comme un ensemble de stimuli ayant un impact sur le comportement du visiteur, nous cherchons à attirer l'attention des concepteurs sur la nécessité de concevoir des activités pourvues de forts stimuli.

Ce schéma indique en effet que les stimuli de l'activité (le sujet, son contenu), s'ils sont suffisamment forts, créeront chez le participant une stimulation, qui se traduira par un comportement réceptif ${ }^{(4)}$. Si le participant répond à la stimulation, il sera alors actif et différents bénéfices pourront être observés : action, créativité, compétences, plaisirs, identité et sociabilité. À l'inverse, si le participant ne répond pas à la stimulation, ce dernier sera passif. La passivité peut toutefois entraîner certains bénéfices relevant du plaisir ou de la compétence. Par exemple, certains parents, tout en étant passifs, peuvent néanmoins prendre du plaisir à voir leur enfant participer à un atelier de création. Autre exemple, on peut enregistrer des connaissances et des savoir-faire sans nécessairement « pratiquer ». La partie droite du schéma montre, pour sa part, qu'il est en revanche fort probable qu'une activité dotée de faibles stimuli entrâne une faible stimulation. Cela se traduira chez le participant par un comportement non-réceptif (5) et l'absence de bénéfices (6).

En définitive, l'intérêt de ce schéma est de signifier aux concepteurs de programmes l'importance de concevoir une activité pourvue de stimuli forts. Notons qu'on voit la force d'un stimulus quand on atteint les objectifs et les bénéfices que l'on s'est fixés au départ. Si le stimulus est faible, le visiteur reproduira le même comportement tout au long de l'activité. À l'inverse, si le stimulus est fort, l'activité modifiera le comportement du participant.

L'élaboration de cet instrument s'est accompagnée de l'identification de six grandes catégories de bénéfices qu'un participant peut potentiellement retirer d'une activité. Ceux-ci ont été regroupés sous l'appellation générique de "bénéfices globaux ». Cela pour deux raisons. Premièrement, afin d'insister sur le fait que ces bénéfices sont susceptibles de se retrouver dans n'importe quel type de programme éducatif et culturel. Deuxièmement, afin de ne pas induire de confusion avec ce que Colette DufresneTassé et André Lefebvre nomment les «bénéfices généraux » et les «bénéfices particuliers » (1996, pp. 109-129). De fait, il ne s'agit pas pour nous de recenser les bénéfices d'une visite mais bien ceux d'une activité, ce qui impose l'emploi d'un vocabulaire distinct pour bien les différencier. 
Nous avons donc identifié six grandes catégories de bénéfices, se déclinant en une série de bénéfices, comme suit :

- Action : qui regroupe les bénéfices «interaction avec l'objet » et «manifestations d'intérêt » (7);

- Créativité : qui rassemble les bénéfices « dépasser les consignes », « développer ses propres idées » et « laisser aller son imagination »;

- Compétences : qui désigne les bénéfices issus de « l'acquisition de connaissances » ou de «savoirfaire »;

- Plaisirs : qui réunit les bénéfices du «bien-être », du « plaisir esthétique », du « plaisir émotionnel » et enfin du «plaisir intellectuel »;

- Identité : qui regroupe les bénéfices de l' « estime de soi », de la «conscience de soi » et de la « construction identitaire »;

- Sociabilité : qui rassemble enfin les bénéfices de «l'interaction », du « lien social », celui de « se familiariser avec le lieu » et de « rompre avec son quotidien ».

Notons que des bénéfices spécifiques peuvent s'ajouter, selon la nature de l'activité considérée, à ces bénéfices globaux. La souplesse de l'instrument que nous avons conçu permet en effet qu'on l'adapte aux particularités de l'activité à évaluer.

\section{Schéma 4 : Instrument permettant d'évaluer} les bénéfices d'une activité

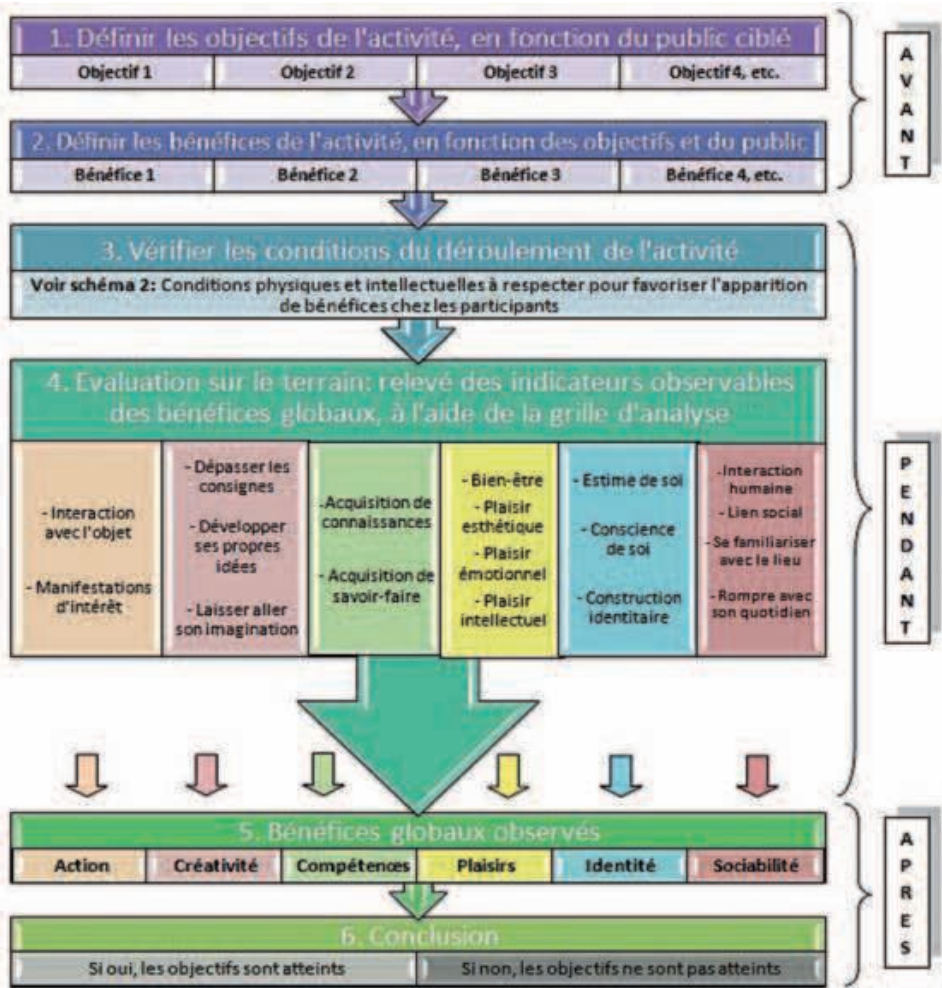

Aboutissement logique des trois schémas précédant, ce dernier schéma permet véritablement l'évaluation sur le terrain d'un programme. Plus exactement, l'évaluation des bénéfices d'une activité passe par six étapes. Avant l'observation sur le terrain, il convient de définir au préalable les objectifs de l'activité (1) et les bénéfices qui en découlent (2). Sur le terrain, il faut vérifier que les conditions physiques et intellectuelles pour favoriser l'apparition de bénéfices chez les participants sont bien remplies ${ }^{(3)}$. On se référera pour ce faire au schéma 2. Il convient également, lorsque l'activité débute, de noter librement tout ce que font et disent les participants. On pourra alors, après l'observation, recourir à une grille des indicateurs observables des bénéfices que nous avons conçue pour quantifier et qualifier les bénéfices observés ${ }^{(4)}$. Après l'observation, grâce à cette grille, il est alors facile de voir quels sont les bénéfices les plus et les moins observés ${ }^{(5)}$. Cela permettra de conclure : si les bénéfices préalablement définis ont été observés, alors les objectifs sont atteints. À l'inverse, si l'on n'a pas observé les bénéfices, les objectifs de l'activité ne sont pas atteints (6). En cas d'échec, il conviendra d'analyser les résultats obtenus au regard de deux éléments. D’une part, l'adéquation entre les objectifs, le public ciblé, la nature de l'activité proposée et les bénéfices escomptés. D’autre part, les conditions de déroulement de l'activité : ont-elles favorisé l'apparition de bénéfices chez les participants ? C'est en procédant de la sorte que l'on pourra repérer les éléments à l'origine des disfonctionnements relevés en termes de bénéfices retirés de l'activité.

Pour faciliter l'observation, nous avons créé une grille recensant les indicateurs observables de nos six catégories de bénéfices. Ces indicateurs peuvent relever à la fois de la communication verbale et de la communication non-verbale. Cette liste d'indicateurs n'est cependant pas exhaustive. Il est loisible à chacun de rajouter des indicateurs observables issus des bénéfices spécifiques à une activité. Par ailleurs, notons que certains de ces indicateurs ne sont pas toujours observables, soit sur le plan de la communication verbale, soit sur celui de la communication non-verbale. Le recours à d'autres outils serait alors nécessaire pour approfondir la recherche. Enfin, certains indicateurs plus difficilement observables ont été assortis d'un exemple, comme on peut le voir dans la grille au verso. 


\begin{tabular}{|c|c|c|c|c|c|c|c|c|c|c|c|}
\hline \multicolumn{12}{|c|}{ Comportement réceptif } \\
\hline \multicolumn{2}{|r|}{ Action } & \multicolumn{2}{|c|}{ Créativité } & \multicolumn{2}{|c|}{ Compétences } & \multicolumn{2}{|c|}{ Plaisirs } & \multicolumn{2}{|l|}{ Identité } & \multicolumn{2}{|c|}{ Sociabilité } \\
\hline $\mathrm{cv}$ & $\mathrm{CNV}$ & $\mathrm{CV}$ & CNV & $\mathrm{CV}$ & $\mathrm{CNV}$ & cv & CNV & $\mathrm{cV}$ & CNV & cv & CNV \\
\hline \multicolumn{2}{|c|}{ Interection aves Fobiet } & \multicolumn{2}{|c|}{ Dépasser les consignes } & \multicolumn{2}{|c|}{ Acquisition de connaissances } & \multicolumn{2}{|c|}{ Bien-être } & \multicolumn{2}{|c|}{ Estime de soi } & \multicolumn{2}{|c|}{ Interaction } \\
\hline & \begin{tabular}{|l|} 
Manipuler lobjet \\
(iprendre, donner. \\
recevoir, etc) et \\
avoir une réponse \\
en retour lex. \\
Gratter une \\
guitare et adapter \\
son geste pour en \\
tirer un son)
\end{tabular} & $\begin{array}{l}\text { Dire qu'on fait } \\
\text { autrement }\end{array}$ & \begin{tabular}{|l|}
$\begin{array}{l}\text { Faire } \\
\text { autrement }\end{array}$ \\
\end{tabular} & $\begin{array}{l}\text { Poser des question } \\
\text { sans lien ou qui vont } \\
\text { au-dela du contenu; } \\
\text { reformuler le contenu } \\
\text { pour se l'approprier; } \\
\text { manifestations de type } \\
\text { "eureka" (ex: ah say } \\
\text { est / ai compris'): } \\
\text { expli quer aux autres }\end{array}$ & $\begin{array}{l}\text { Opiner } \\
\text { (attention, ceia } \\
\text { n'est pas } \\
\text { toujours un } \\
\text { bon indicateur } \\
\text { de la } \\
\text { comprehension } \\
\text { rélle du sujet) }\end{array}$ & \begin{tabular}{|l|} 
Manifestations \\
verbaies qui \\
traduit un bien- \\
ëtre phys que \\
et \\
psychoiogique \\
(ex: Qu'est-ce \\
qu'on est bien \\
laill)
\end{tabular} & $\begin{array}{l}\text { Confort } \\
\text { phys ique et } \\
\text { psychologique: } \\
\text { etre detendu }\end{array}$ & $\begin{array}{l}\text { Expressions verbales de } \\
\text { "auto-satisfaction (ex. } \\
\text { 'Eh, je suis plutot doue r) }\end{array}$ & $\begin{array}{l}\text { Sentiment } \\
\text { d'auto- } \\
\text { efficacité: se } \\
\text { sentir } \\
\text { suffisament } \\
\text { competent pour } \\
\text { faire; postures } \\
\text { de fierte (ex. } \\
\text { bomber le torse) }\end{array}$ & $\begin{array}{l}\text { Poser des questions, } \\
\text { yrépondre; attirer } \\
\text { 'rattention; } \\
\text { reconfortec; } \\
\text { encourager; feliciter; } \\
\text { remercier; } \\
\text { complimenter; } \\
\text { discuter }\end{array}$ & \begin{tabular}{|l|} 
S'échanger le \\
matériel; stimuler \\
sensoriellement \\
'rautre; montrer \\
comment faire; \\
laisser faire; \\
diriger; caresser; \\
embrasser; \\
s'amuser ensemble \\
\end{tabular} \\
\hline \multicolumn{2}{|c|}{ Manifestations d'intérêt } & \multicolumn{2}{|c|}{ Développer ses propres idée } & \multicolumn{2}{|c|}{ Acquisition de savoir-faire } & \multicolumn{2}{|c|}{ Plaisir esthétique } & \multicolumn{2}{|c|}{ Conscience de soi } & \multicolumn{2}{|c|}{$\begin{array}{l}1 \\
\text { Lien social } \\
\end{array}$} \\
\hline $\begin{array}{l}\text { lice un } \\
\text { texte a } \\
\text { voix } \\
\text { haute }\end{array}$ & $\begin{array}{l}\text { lire dans se tette } \\
\text { regarder } \\
\text { attentivement. } \\
\text { ecouter } \\
\text { attentivement. }\end{array}$ & \begin{tabular}{|l|} 
S'approprier le \\
contenu en le \\
reformulant, \\
pour lui \\
donner une \\
dimension plus \\
personnelle \\
\end{tabular} & \begin{tabular}{|l|} 
Faire \\
autrement \\
mais de \\
maniére \\
plus \\
aboutie
\end{tabular} & $\begin{array}{l}\text { Expl lquer ce qu'll faut } \\
\text { faire; manifestation de } \\
\text { type "eureka" lex: ah } \\
\text { d"accord jai compris } \\
\text { qu'li fout faire comme } \\
\text { co) }\end{array}$ & $\begin{array}{l}\text { Mettre en } \\
\text { pratique ce quil } \\
\text { a été expliqué. } \\
\text { faire } \\
\text { manue llement }\end{array}$ & \begin{tabular}{|l|} 
Manifestations \\
verbales du \\
type "c'est \\
beaul"
\end{tabular} & & 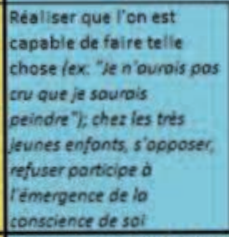 & & $\begin{array}{l}\text { Poser des questions, } \\
\text { v répondre; attirer } \\
\text { rattention; } \\
\text { reconforter; } \\
\text { encourager, feliciter: } \\
\text { remercier; } \\
\text { complimenter; } \\
\text { discuter }\end{array}$ & $\begin{array}{l}\text { S'echanger le } \\
\text { matériel; stimuler } \\
\text { sensoriellement } \\
\text { I'autre; montrer } \\
\text { comment faire; } \\
\text { laisser faire; } \\
\text { diriger; caresser; } \\
\text { embrasser; } \\
\text { s'amuser ensemble } \\
\end{array}$ \\
\hline & & \multicolumn{2}{|c|}{ Laisser aller son imagination } & & & \multicolumn{2}{|c|}{ Plaisir émotionnel } & \multicolumn{2}{|c|}{ Sonstruction identitaire } & \multicolumn{2}{|c|}{ Se famillariser avec le lieu } \\
\hline & & \begin{tabular}{|l|} 
imagination \\
créatrice lex: \\
inventer une \\
histoire): \\
imagination \\
reproductrice \\
(ex: foire appel \\
¿d ses souvenirs \\
\end{tabular} & \begin{tabular}{|l} 
Etre tries \\
spontané \\
dans la \\
création
\end{tabular} & & & \begin{tabular}{|l|} 
Rires, \\
gazouilils, cris \\
d'excitation, \\
mots doux. \\
chanter
\end{tabular} & & $\begin{array}{l}\text { S'opposer dans un sens } \\
\text { constructif, chez les } \\
\text { adolescents notamment } \\
\text { iex. Mol je ne suis pos } \\
\text { d' occord parce-que.-. }\end{array}$ & & \begin{tabular}{|l} 
Manifestations \\
verbales de la \\
familliarisation au \\
lieu lex: "Viens, je \\
vois te montrer où se \\
passe lactivite"l
\end{tabular} & $\begin{array}{l}\text { Ne pas se sentir } \\
\text { perdu; avoir des } \\
\text { points de repères } \\
\text { dans lespace et } \\
\text { dans le temps qui } \\
\text { font que Pon est a } \\
\text { Paise dans cet } \\
\text { environnement. } \\
\end{array}$ \\
\hline & & & & & & \multicolumn{2}{|c|}{ Plaisir intellectuel } & & & \multicolumn{2}{|c|}{ Rompre avec son quotidien } \\
\hline & & & & & & \begin{tabular}{|l|} 
Manifester \\
verbalement ie \\
plaisir retiré \\
d'avoir appris, \\
compris des \\
choses \\
\end{tabular} & & & & \begin{tabular}{|l} 
Exprimer le \\
changement induit \\
par la venue lex: "Co \\
change du boulot/de \\
ce qu'on fait à lo \\
maison") \\
\end{tabular} & \\
\hline
\end{tabular}

\section{Validation de l'instrument de recherche}

Il est à noter que cet instrument de recherche a été validé sur le plan théorique par plusieurs professionnels de musées et de l'éducation. Dans un premier temps, nous l'avons soumis à François Bowen, professeur au département de Psychopédagogie et Andragogie, et vice-doyen aux études supérieures et à la recherche, de la faculté des Sciences de l'Éducation de l'université de Montréal. Dans un second temps, cet instrument a reçu l'aval de deux chercheuses en muséologie associées à l'université de Montréal : Marie-Clarté O'Neill et Colette Dufresne-Tassé.

Fortes des conseils prodigués par ces professionnels, nous avons ensuite testé cet instrument sur le terrain à deux reprises. D'abord avec une activité intitulée Parents-Poupons, proposée par le musée McCord d'Histoire canadienne, puis avec un atelier de création littéraire, destiné à des adolescents et adultes, se déroulant dans une bibliothèque à Laval. $\mathrm{Au}$ terme de ces deux essais, l'emploi de notre instrument d'évaluation s'est révélé concluant.

\section{Synthèse : atouts et limites de l'instrument}

En définitive, l'instrument de recherche que nous avons élaboré pour évaluer les bénéfices d'un programme présente de nombreux atouts.
Premièrement, les schémas en question rendent compte du contexte dans lequel se déroulent l'activité et son évaluation. Or, c'est bien souvent une présentation de la situation d'un point de vue environnemental qui fait défaut aux évaluations de programmes. Cet instrument permet donc de lier les objectifs d'un programme et les bénéfices escomptés aux résultats obtenus, mais sans en occulter le contexte. Procéder de la sorte présente un double avantage. D'une part, cela permet de revisiter l'activité : ses objectifs, son contenu, mais aussi son déroulement, ses applications pour comprendre pourquoi on est arrivé à tel résultat. D'autre part, cela donne une prise sur l'activité, ce qui permet de la piloter voire de l'ajuster dans son déroulement.

Deuxièmement, l'instrument différencie les objectifs des bénéfices et oblige en cela, à la fois le concepteur et l'évaluateur d'un programme, à en avoir une conscience articulée. De fait, nous avons considéré que les bénéfices ne doivent pas être confondus avec les objectifs, car s'ils en découlent ils vont bien au-delà des objectifs (8).

Troisièmement, le schéma 4 permet de savoir rapidement et avec peu de moyens si une activité génère les bénéfices escomptés, de les quantifier et de les qualifier. Ce schéma est adaptable à tout type d'activité, et ce quel que soit le public concerné, en fonction des objectifs et des bénéfices du programme à évaluer. Il 
est donc d'une grande souplesse dans son application et son usage. Enfin, ce dernier schéma autorise plusieurs niveaux de lecture selon les besoins de l'institution. On peut se contenter d'une analyse quantitative, qui comptabilise les bénéfices. Ou mener une étude plus approfondie autour de la nature de la communication, des indicateurs observables relevés ou des participants eux-mêmes.

Pour autant, la principale limite de cet instrument réside dans le fait que certains bénéfices sont difficilement observables. L'observation est dans certains cas insuffisante, et il peut être nécessaire de recourir à d'autres outils, comme l'entretien, pour investiguer certains bénéfices relevant de l'identité ou des compétences.

Au final, rappelons que cet instrument de recherche a pour vocation de synthétiser les connaissances que l'on peut avoir sur l'évaluation d'un programme, et d'aider à sa réalisation et à son évaluation. L'instrument que nous avons développé cherche ainsi à décortiquer chaque étape d'une activité, depuis sa création jusquà sa réalisation, pour aider à une meilleure compréhension du processus et des résultats obtenus. Surtout, c'est un dispositif à la fois peu contraignant et qui nécessite peu de moyens humains et financiers à mettre en œuvre pour une institution qui voudrait savoir si l'un de ses programmes génère les bénéfices qu'elle en attend. Pour toutes ces raisons, cet instrument peut être d'une réelle utilité pour les professionnels, soit en amont de la création d'un programme, soit en aval pour y évaluer les bénéfices.

\section{Notes}

(1) À l'exception du Guide de planification et d'évaluation des programmes éducatifs conçu par Michel Allard, Marie-Claude Larouche, Anik Meunier et Pierre Thibodeau, dont nous avons eu connaissance après la création de notre outil.

(2) Cette recherche s'inscrit dans le cadre d'un stage réalisé au sein du Musée McCord d'histoire canadienne à Montréal, de juillet à octobre 2008, en partenariat avec l'Ecole du Louvre et l'Université de Montréal.

(3) Site Internet de l'École du Louvre : www.ecoledulouvre.fr/ pdf/pdf_doc/prog02.pdf.

(4) Par comportement « réceptif », on entend être en phase avec l'activité. Cela peut se traduire durant l'activité par un certain nombre de comportements relevant de la communication verbale ou non-verbale, tels que rire, s'amuser, écouter...

(5) À l'inverse, le comportement non-réceptif induit que le participant n'est pas en phase avec l'activité. Cela s'observe durant l'activité au travers d'un certain nombre de comportements relevant de la communication verbale ou non-verbale, tels que crier, pleurer, bouder, critiquer...

(6) Il pourrait à ce titre être intéressant de mener une étude approfondie sur le sujet, afin de voir si - comme nous le croyons - un comportement non-réceptif n'entraîne aucun bénéfice. On gagnerait aussi à mieux appréhender les conditions d'apparition d'un stimulus fort par rapport à un stimulus faible.

(7) Nous avions, dans un premier temps, inclus dans le bénéfice global «action " la manipulation d'objets et la stimulation sensorielle. Nous les en avons ôté après que Colette Dufresne-Tassé ait attiré notre attention sur le fait que ceux-ci n'étaient pas des bénéfices en soi, mais plutôt la source de bénéfices.

(8) Un exemple simple permet de le comprendre. Prenons le cas d'une personne souhaitant apprendre à jouer d'un instrument. Son objectif sera de savoir se servir dudit instrument. Ses bénéfices seront en revanche bien plus vastes : être capable de déchiffrer tout type de partition, de jouer un air entendu, de connaître l'histoire de la musique avec les grands compositeurs et interprètes...

\section{Bibliographie}

Allard, M., Larouche, M.-C., Meunier, A. et Thibodeau, P. Guide de planification et d'évaluation des programmes éducatifs. Lieux historiques et autres institutions muséales. Québec : Les Éditions Logiques, 1998, 239 p.

Allard, M. et Larouche M.-C. Un modèle théorique d'évaluation des programmes éducatifs mis en œuvre dans les lieux historiques, in Lefebvre, B. et Allard, M. Le musée au service de la personne. Québec : UQAM, 1999, pp. 303-318.

Dufresne-Tassé, C., Lapointe, T. et Lefebvre, H. Exploratory study of the benefits of a visit to the museum, The Canadian Journal for the Study of Adult Education, VII, 1-19, 1993, pp. 1-28.

Dufresne-Tassé, C. et Lefebvre, A. Psychologie du visiteur de musée. Contribution à l'éducation des adultes en milieu muséal. Québec : Cahiers du Québec, collection Psychopédagogie, 1996, pp. 109-129.

Lefebvre, H. Les bénéfices retirés par la personne retraitée lors de la visite au musée, in Lefebvre, B. et Allard, M. Le Musée un projet éducatif. Montréal : Les Éditions Logiques, 1996, pp. 193-205.

Packer, J. Beyond Learning : Exploring Visitor's Perceptions of the Value and Benefits of Museums Experiences, Curator, volume 51, $n^{\circ} 1$, janvier 2008, pp. 33-54.

Benharkate, S. et Degain, J. Création d'instruments de recherche pour analyser et évaluer les " bénéfices » d'une activité offerte par le musée McCord d'histoire canadienne. Mémoire de Maîtrise en Muséologie de l'université de Montréal juillet-septembre 2008, 130 p. (consultable à l'École du Louvre et à l'université de Montréal) 